\title{
Including Both Costs and Effects - The Challenge of Using Cost-Effectiveness Data in National-Level Policy-Making: A Response to Recent Commentaries
}

\author{
Nathalie Eckard ${ }^{1 *}$, Magnus Janzon ${ }^{2}$, Lars-Åke Levin ${ }^{1}$
}

\author{
*Correspondence to: Nathalie Eckard, Email: nathalie.eckard@liu.se \\ Copyright: (๑) 2015 by Kerman University of Medical Sciences \\ Citation: Eckard N, Janzon M, Levin LA. Including both costs and effects - \\ the challenge of using cost-effectiveness data in national-level policy-making: a \\ response to recent commentaries. Int J Health Policy Manag. 2015;4(8):565-566. \\ doi:10.15171/ijhpm.2015.123 \\ Received: 23 June 2015, Accepted: 3 July 2015, ePublished: 8 July 2015
}

$I^{2}$

ncorporating cost-effectiveness data in the priority-setting decisions is a distinguishing feature in the Swedish national guidelines. This differentiates the Swedish guidelines from its European and American counterparts. The broader perspective is aimed at facilitating explicit resource allocations and priority-setting in healthcare, all in accordance with the three guiding ethical principles stated in the Health and Medical Services Act. ${ }^{1}$ The guidelines consist of recommendations in the form of a priority-setting decisions produced by a Priority-Setting Group (PSG) and the work process involves appraising the evidence used as decision support (including cost-effectiveness).

In our article "Use of cost-effectiveness data in priority-setting decisions: experiences from the national guidelines for heart diseases in Sweden" we expressed that "economic evidence should be viewed as part of the evidence-based knowledge "package." It is not our intention to argue that healthcare priority-setting should be based solely on cost-effectiveness data. The use of cost-effectiveness data represents one of many factors that play a role in health policy decision-making." Despite the "success" of using cost-effectiveness data in decision-making as commented by Williams and Bryan ${ }^{3}$ and also being "a wonderful exception to this rule" as commented by Erntoft, ${ }^{4}$ it was well-observed that cost-effectiveness data was lacking as decision support in the majority of cases. The study design did not use quantitative approach, to explore the use of cost-effectiveness data in decision-making. Instead, we have given examples of situations when cost-effectiveness arguments impacted decisions taken and was used in the PSG deliberations.

There is a need to recognise the contextual elements in decision-making, as Williams and Bryan put it, and highlight institutional factors and incentives to employ use of costeffectiveness analyses in decision-making. ${ }^{5}$ The linear and simplistic goal of arriving at collective agreement is presented as an ideal for how to work with evidence-based policy (EBP). The work on producing as well as implementing guidelines, is not as easily achieved as is preferably framed in the idealistic rational policy-making model. In practice, all steps-when evidence is produced, when evidence-informed guidelines in the form of policy documents are produced and when these guidelines are interpreted and used in practice - involve negotiations and collective sense-making. ${ }^{1,6}$

Our case study of the national guidelines constitute nationallevel policy decision-making. We are aware of the fact that, empirical research has shown that healthcare decisionmakers at the local level have not yet accepted the use of costeffectiveness evidence in their decision-making to the same extent as at the national level concerning pharmaceuticals. ${ }^{7}$ One reason for this is that affordability is often a pressing issue at local level decision-making and outcomes are seldom viewed in relation to cost. At a national policy level in Sweden, a societal perspective including both costs and effects is often applied. Though, the commentary by Erntoft concludes that certain conditions need to be fulfilled to enable the use of costeffectiveness data in real life. ${ }^{4}$ These include lack of budget restriction, strategies to handle uncertainty, transparency, legitimising health economics through a health economist attending the PSG deliberations, and clear instructions (use of templates) and nicely fits the results of our study.

Cost-effectiveness data played an important role in cases of greater uncertainty and ambiguity. Economic arguments clearly became a part of the PSG deliberation when the group members had difficulty in reaching agreement. As observed by Youngkong, ${ }^{8}$ setting healthcare priorities is not likely to succeed without considering deliberative processes among concerned stakeholders, justifying their reasons to maintain authority in decision-making. The use of cost-effectiveness data may therefore be regarded as a format for moving the work process forward, avoiding blocks in discussion, and viewed as a "solution" to solve the task of making prioritysetting decisions. Using cost-effectiveness arguments was also used to structure the discussions and interestingly was not challenged to the same extent as clinical evidence. Economic arguments was used as a fine-tuning instrument and a counterweight for dichotomization.

Ethical issues

Not applicable.

Competing interests

Authors declare that they have no competing interests.

${ }^{1}$ Division of Health Care Analysis, Department of Medical and Health Sciences, Linköping University, Linköping, Sweden. ${ }^{2}$ Department of Cardiology and Department of Medicine and Health Sciences, Linköping University, Linköping, Sweden. 
Authors' contributions

Each author has contributed to the writing of this correspondence. Each author approves the version being submitted and certifies that the contents has not been published or is being considered for publication elsewhere.

\section{References}

1. Health and Medical Service Act, SFS 1982:763 (1982).

2. Eckard N, Janzon M, Levin LA. Use of cost-effectiveness data in priority setting decisions: experiences from the national guidelines for heart diseases in Sweden. Int $J$ Health Policy Manag. 2014;3(6):323-332. doi:10.15171/ijhpm.2014.105

3. Williams I, Bryan S. Lonely at the top and stuck in the middle? The ongoing challenge of using cost-effectiveness information in priority setting. Int J Health Policy Manag. 2014;4(3):185-187. doi:10.15171/ijhpm.2015.32

4. Erntoft $\mathrm{S}$. The use (or rather non-use) of cost-effectiveness data in priority setting decisions - are we underestimating the barriers to using health economics in real world priority setting decisions?
Int J Health Policy Manag. 2015;4(3):181-183. doi:10.15171/ ijhpm.2015.28

5. Williams I. Institutions, cost-effectiveness analysis and health care rationing: the example of health care coverage in the English National Health Service. Policy Polit. 2013;41(2):223239. doi:10.1332/030557312x655477

6. Nedlund AC, Garpenby P. Puzzling about problems: the ambiguous search for an evidence-based strategy for handling influx of health technology. Policy Sci. 2014;47(4):367-386. doi:10.1007/s11077-014-9198-1

7. Ernoft S. The Use of Health Economic Evaluations in Pharmaceutical Priority Setting. The Case of Sweden [dissertation]. Lund: Lund Business Press, Lund Institute of Economic Research, Lund University; 2010

8. Youngkong S. Incorporating cost-effectiveness data in a fair process for priority setting efforts. Int $\mathrm{J}$ Health Policy Manag. 2015;4(7):483-485. doi:10.15171/ijhpm.2015.81 